\title{
Link performance evaluation of Uplink Precoded Multiuser MIMO-NOMA system for 5G Communication Networks
}

\author{
Kishore K R*, Pagadala Dinesh Kumar \\ Research Engineer \\ Centre for Development of Telematics \\ Bangalore, India \\ *kishorekr@cdot.in
}

\begin{abstract}
Non orthogonal Multiple Access (NOMA) is a promising candidate for future radio access. Due to the characteristic property of NOMA to multiplex users in time, frequency and power domains, it can achieve capacity gains superior to OFDMA (orthogonal frequency division multiple access). However, being a multi-carrier modulation scheme, NOMA suffers from high peak to average power ratio (PAPR) in addition to the interference at the receiver terminal due to its non-orthogonal nature. In this paper we analyses the link level performance of $2 \times 2$ MIMO (Multiple Input Multiple Output) NOMA uplink system with Hadamard Transform Precoding (HTP) over Rayleigh fading channel model. The HTP NOMA enhances the PAPR performance by $3 \mathrm{~dB}$ over the non precoded NOMA.
\end{abstract}

Keywords-5G, NOMA, MIMO, PAPR.

\section{INTRODUCTION}

One of the hurdles that is faced by the advent of fifthgeneration cellular networks $(5 \mathrm{G})$ is the explosive number of users and things that gets connected to the network. The design of the Radio access network (RAN) and its multiple access technique play a pivotal role in the enhancing the network capacity. The orthogonal multiple access (OMA) is impotent to cater future radio access demands which pave the way for the non-orthogonal multiple access schemes (NOMA) [1-4].

3GPP (Third generation partnership project) has recognized the potential and necessity of NOMA for future radio access that it had been a study item in Release 13 and work item in Release 14 for 4G-LTE technology downlink [12]. For $5 \mathrm{G}$ standardization, NOMA has been extensively studied in Release 16 and summarized in the technical report 38.812 for uplink scenario instead of downlink, in order to minimize the hardware complexity at user equipment (UE) [1]. NOMA offers improved spectral efficiency (SE), higher sum rate, higher cell-edge throughput, relaxed channel feedback, low transmission latency and grant free access (no scheduling request from users to base station) [2- 4].

NOMA can be broadly classified into power domain NOMA(PD-NOMA) and code domain NOMA(CD-NOMA). The PD- NOMA attains multiplexing in power domain by taking into account the geographical distribution of users (path loss) within a cell and to opportunistically allocate power among the multiplexed users on same time, frequency resources. The CD- NOMA achieves multiplexing in code domain, reminiscent of the classical code division multiple access (CDMA) systems, using user-specific spreading sequences that include non-orthogonal cross correlation sequences of low correlation coefficient or sparse sequences [2]. Thanks to the superposition coding (SC) technique at the transmitter and successive interference cancellation (SIC) at the receiver for being the success behind the realisation of NOMA. In SC technique, single transmitter communicates to multiple users by encoding data specific to each receiver [2]. The SC encoded signal constellation is detected using a SIC receiver. In a SIC receiver, strongest user signal is decoded, treating the other user signal as an interferer, but the latter is then decoded with the knowledge of the former. The SIC decoding involves hardware complexity for PD-NOMA and attracts much of research interest in NOMA [2].

The combination of NOMA with MIMO has been widely investigated in the literature [5- 7]. Zhiguo et al., proposes precoding and detection schemes in downlink scenario for MIMO-NOMA systems [5]. Anass et al., experimentally validates the concept of MIMO-NOMA through field trials in LTE downlink scenario under realistic conditions. The experimental trials using $2 \times 2$ open-loop MIMO and 4x2 closed-loop MIMO were performed for three users in outdoor environment which claim that the $4 \times 2$ closedloop MIMO-NOMA can provide a maximum of 2.3 times more gain compared to SU-MIMO [6]. MIMO-NOMA for uplink has been investigated for mm wave channel models utilising the concept of hybrid beamforming to enhance the energy efficiency. The authors claim that the proposed scheme achieve better sum rate to improve the performance gap of MIMO-OMA with that of MIMO-NOMA [7].

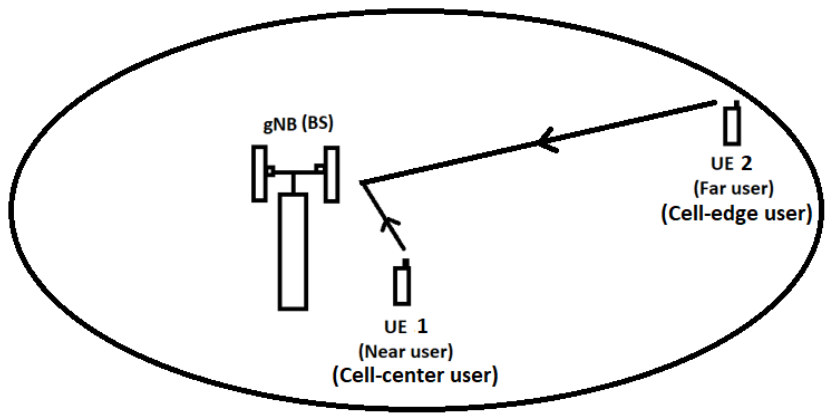

Figure 1. Typical uplink scenario with two users and single base station

NOMA opens up immense opportunities due to its high spectral efficiency, but suffers from non-appreciable energy efficiency. The NOMA thrives on non-constant envelope, multicarrier modulation scheme such as orthogonal frequency division multiplexing (OFDM), which restrains high peak to average power ratio (PAPR) due to the in-phase addition of the subcarriers at the output of IFFT. The penalty of high PAPR is paid as higher power back off at the power amplifier and stringent linearity requirements to trace the varying waveform envelope. To combat this scenario, precoding matrices has been proposed in literature to reduce 
the autocorrelation of the modulation symbols prior to the inverse fast Fourier transform (IFFT) operation [8].

In this paper, precoded PD-NOMA with multiuser MIMO (MU-MIMO) is investigated for uplink consisting of two users and a base station (gNB) as shown in figure 1 where the cell-center near user and cell-edge far user is paired. Followed by the introductory note at section I, section II describes a brief theoretical overview with inevitable mathematical interpretations to conceive the system model depicted in section III. The effect of precoded matrix on PAPR performance is described in section IV. The bit error performance of 2x2 MIMO-NOMA link level simulation is discussed in section IV. The simulations and discussions embodied in the preceded sections are concluded in section VI. Henceforth, this paper refers to PD-NOMA simply by NOMA.

\section{THEORY: 2X2 MIMO NOMA SYSTEM}

In this section, mathematical overview of the system is discussed.

\section{A) Superposition coding $(S C)$}

In uplink NOMA, the paired users will be allotted a normalized power scaling factor by the base station based on the channel characteristics of each user.

Consider SC encoding for two users, $\mathrm{f} 1:\{0,1\}\left\llcorner^{2 T R 1}\right\lrcorner$ $\rightarrow C^{T}$ and f2: $\{0,1\}\left\llcorner^{2 T R 2}\right\lrcorner \rightarrow C^{T}$. Here ' $R 1$ ' and ' $R 2$ ' denote the transmission rates of user 1 and user 2 , respectively, each of block length ' $T$ '. ' $C$ ' denotes the code library and $L \cdot\lrcorner$ is the floor operator. conditions: -

1. The elements of HT precoding Matrix consist of \pm 1 .

2. The HT precoding matrix should be a non-singular and orthogonal.

The above conditions ensure that all output symbols deliver same amount of information for every input, and the original data must be recovered at the receiver side [8].

C) Root raised cosine (RRC) filtering

Root raised cosine (RRC) filtering is a pulse shaping technique to minimize the ISI and to limit the excess bandwidth. The RRC filter is defined as follows

$$
r(t)=\frac{\sin \left(\frac{\pi t}{T}(1-\alpha)+4 \alpha \frac{t}{T} \cos \left(\frac{\pi t}{T}(1+\alpha)\right.\right.}{\frac{\pi t}{T}\left(1-\frac{16 \alpha^{2} t^{2}}{T^{2}}\right)}
$$

where, ' $\alpha$ ' denotes the roll off factor and ' $T$ ' is the symbol duration [8].

\section{D) Peak to average power ratio (PAPR)}

Peak to average power ratio (PAPR) of the signal, $s(t)$ is defined as

$$
\text { PAPR }=\frac{\max |s(t)|^{2} ; 0 \leq t \leq N T}{\frac{1}{N T} \int_{0}^{N T}|s(t)|^{2} d t}
$$

Where ' $N$ ' denotes the total number of subcarriers and ' $T$ ' is the symbol duration [8].

E) Successive interference cancellation (SIC)

SIC is feasible only when there is a difference in the power allocation among the signals of interest. The signals are ordered in the order of their signal strengths prior to SIC. Signal with high signal power is detected first, subtract it from the received SC signal, and isolate the weaker one from the

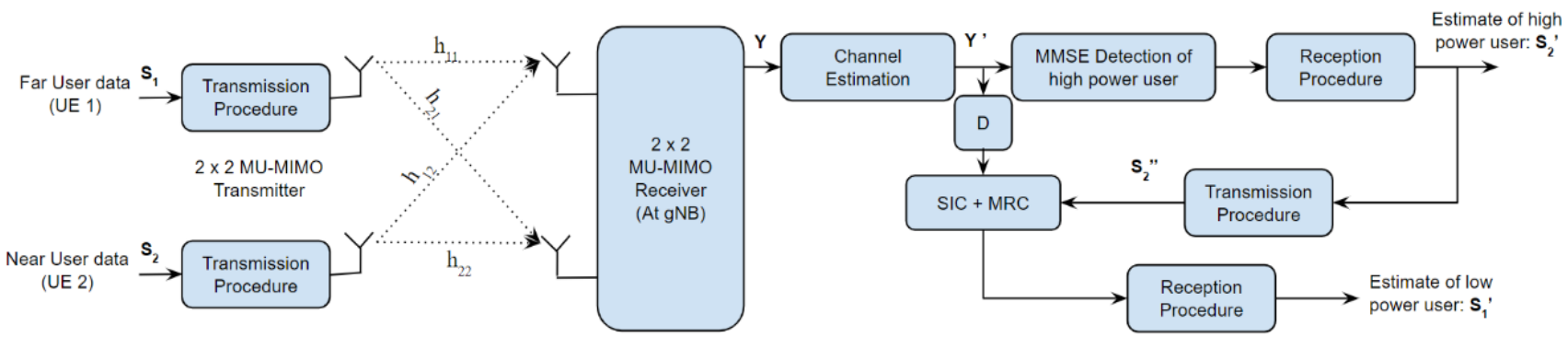

Figure 2: System Model of 2 × 2 MIMO-NOMA System

For the two-user uplink case as shown in figure 2 , let ' $\beta_{1}$ ', ' $\beta_{2}$ ' be the power allocation coefficients for user 1 and user 2 respectively such that $\beta 1+\beta 2=1$. Then the $\mathrm{SC}$ signal at the channel input at $n^{\text {th }}$ instant can be regarded as

$$
X(n)=\sqrt{P \beta_{1}} S_{1}(n)+\sqrt{P \beta_{2}} S_{2}(n)
$$

where ' $P$ ' represents the total power allocated to the user pair and ' $S_{1}$ ' and ' $S_{2}$ ' represents the constellation symbols of the user 1 and user 2 respectively [2].

\section{B) Hadamard Transform (HT)}

The Hadamard Transform (HT) is a less complex, recursive transform kernel implementable using a butterfly structure similar to FFT.

HT can be written as: -

$$
\begin{gathered}
H_{1}=[1] \\
H_{2}=\frac{1}{\sqrt{2}}\left[\begin{array}{cc}
1 & 1 \\
1 & -1
\end{array}\right] \\
H_{2 N}=\frac{1}{\sqrt{2} N}\left[\begin{array}{cc}
H_{N} & H_{N} \\
H_{N} & H_{N}^{-1}
\end{array}\right]
\end{gathered}
$$

where, $H_{N}{ }^{-1}$ defines a binary complement of $H_{N}$. The HT precoding matrix should fulfil the following residue.

For a two-user NOMA uplink, SIC is performed at the base station (BS). The received signal at the BS for $2 \times 2$ MIMONOMA can be represented as

$$
\left[\begin{array}{l}
y_{1} \\
y_{2}
\end{array}\right]=\left[\begin{array}{ll}
h_{11} & h_{12} \\
h_{21} & h_{22}
\end{array}\right]\left[\begin{array}{cc}
\sqrt{P \beta_{1}} & 0 \\
0 & \sqrt{P \beta_{2}}
\end{array}\right]\left[\begin{array}{l}
S_{1} \\
S_{2}
\end{array}\right]+\left[\begin{array}{cc}
\sigma_{w}^{2} & 0 \\
0 & \sigma_{w}^{2}
\end{array}\right]
$$

Where ' $y_{i}$ ' is the received signal vector at ' $i$ 't ' receiver, ' $h_{i j}$ ' denotes the channel path gain at the ' $i$ th' receiver for the signal transmitted from ' $j^{t h}$, transmitter when the signal traverses through the Rayleigh channel. ' $\sigma_{w}^{2}$ ' denotes the additive white gaussian noise (AWGN) [5].

\section{SYSTEM MODEL: 2X2 MIMO NOMA SYSTEM}

The precoded MU-MIMO NOMA system model consists of two users multiplexed in power domain communicating with a $5 \mathrm{G}$ base station (gNB) in an uplink scenario as shown in figure 2. The users are paired based upon their channel path gains and allotted their respective transmit power levels using the power allocation algorithms prior to uplink connection establishment [2]. 


\section{A) Transmission procedures}

The physical layer (PHY) processing of the two users are identical except for the power allocation factor as shown in figure3(a). The arbitrary data generated presuming as medium access control (MAC) layer input to the PHY is encoded by polar coding according to the 3GPP PHY standards [9]. The encoded data is passed on to 16-QAM modulation mapper resulting in complex symbols. In order to reduce the autocorrelation of the generated symbols, the data is precoded using Hadamard matrix. The symbols which are mapped to subcarriers in localized mode is passed onto pulse shaping filter to mitigate inter symbol interference (ISI) and to limit excess bandwidth. Root raised cosine (RRC) filter is employed with a roll off factor of 0.22 and an oversampling factor of eight [8]. retransmission of the high-power user. After cancellation of high-power user by SIC, the 2x2 MIMO effectively deduces to $1 \times 2$ MIMO. Now using maximal ratio combing (MRC) technique, the multipath component of low power user is combined by the virtue of diversity reception. Further, the reception procedures described in figure $3(\mathrm{~b})$ is carried out for the estimation of low power user at $\mathrm{gNB}$.

\section{PAPR PERFORMANCE}

The effect of precoding can be understood by plotting the complementary cumulative distribution function $(\mathrm{CCDF})$ as shown in the figure 4.

The CCDF portrays the probability that the PAPR of the subcarriers exceed a reference PAPR $\left(\mathrm{PAPR}_{0}\right)$. The PAPR performance is evaluated using 323 OFDM symbols

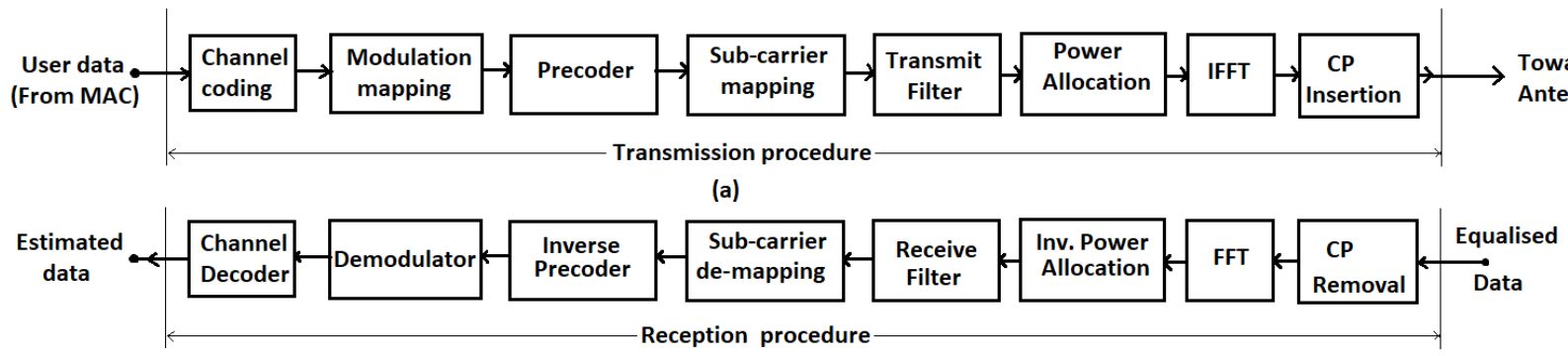

(b)

Fig. 3 (a) Transmission procedure (b) Reception procedure.

The frequency domain samples are converted to time domain OFDM symbols after the IFFT operation. Cyclic prefix of quarter the symbol duration is appended to the generated OFDM symbols which act as a guard band to prevent ISI, hence combat the delay spread of the multipath channel.

The multi user data from the two users interfere in the channel, since the signal occupies the same time and frequency resources. Hence at the $\mathrm{gNB}$, noise and interference corrupted superimposed users need to be speared carefully by complex multi user detection (MUD)-SIC implementations.

B) Reception procedures

The data transmitted by the MU- MIMO transmitter is passed through a Rayleigh channel fading model with AWGN. At the gNB, we assume a 2x2 MIMO receiver with perfect channel state information.

The minimum mean square equalization (MMSE) is performed immediately after the reception which enables the detection of high-power user (user-2) after the reception procedures as shown in figure 3(b). The equalized data output of the particular user undergoes CP removal, fast Fourier transform (FFT) to convert the time domain symbols to frequency domain data samples which are scaled by the inverse power coefficients. The samples are passed on to the inverse filter followed by subcarrier de-mapping. The inverse Hadamard matrix is performed (since, $H . H^{-1}=I$ ) before passing onto the modulation de-mapper and channel decoding sections to extract the estimated data samples.

In order to estimate the low power user (user-1), the component of the signal power from the high-power user should be cancelled out from the equalized received signal vector ' $Y$ ' using SIC. For this the high-power estimated signal, is 'retransmitted' within the receiver chain as shown in figure 2. For faithful cancellation, delay element ' $D$ ' is introduced which is the total delay associated with the estimation and consisting 12 subcarriers each according to the transmission procedure shown in figure 3(a).

\section{Effect of precoding in PAPR performance of NOMA}

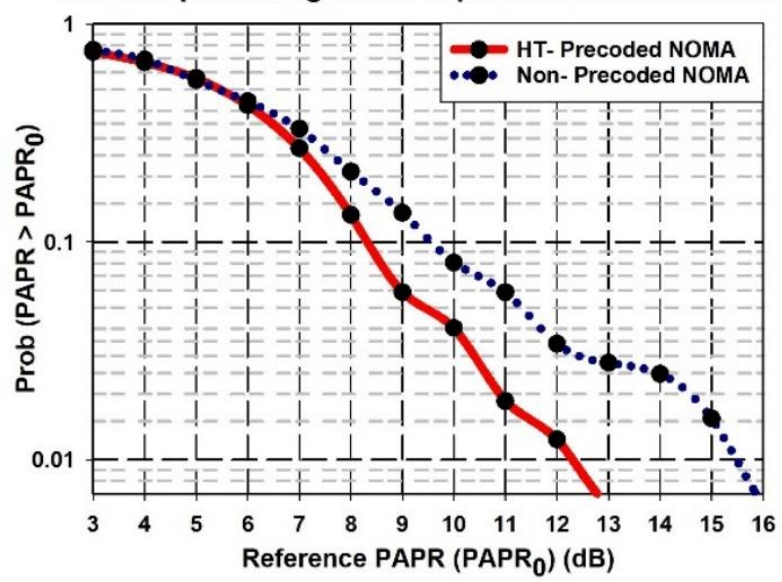

Figure 4. CCDF plot depicting the PAPR performance in HT-precoded NOMA and non-precoded NOMA.

It is evident that the at CCDF clip rate of $10^{-2}$, the precoded NOMA has a PAPR of $12.34 \mathrm{~dB}$ while that of the nonprecoded NOMA is $15.45 \mathrm{~dB}$. Hence the precoding improves the PAPR performance by more than $3 \mathrm{~dB}$.

\section{LINK LEVEL PERFORMANCE}

The link level MU- MIMO-NOMA is configured as per the simulation parameters given in table $\mathrm{I}$. The performance is evaluated using the polar coding technique according to 3GPP specifications for 5G NR [9]. To enhance the link reliability and to avoid the inter-block interference (IBI) owing to the worst-case delay spread of channel, $2 \mathrm{x}$ number of null carriers are padded for the given number of subcarriers, which is not a mandate for computer simulations. The link performance is evaluated by analyzing the bit errors 
encountered by the two users, each modulated by 16-QAM for various signal to noise ratio (SNR). The bit error- SNR relationship for detection of high-power user by MMSE and low power user by SIC for polar coding with a code rate of $7 / 64$ is obtained as shown in figure 5.

\section{Bit error performance of NOMA for two user pairing}

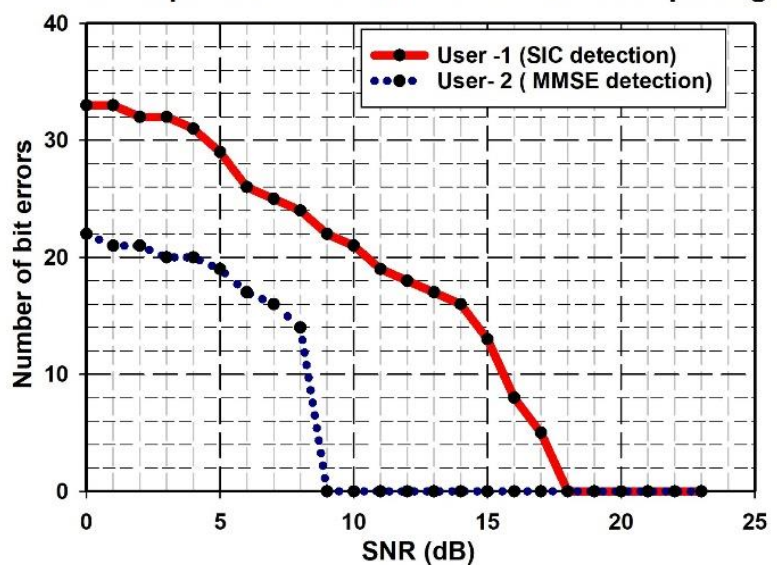

Figure 5. Bit errors obtained for the two users for different SNR values.

It is explicit that the high-power user can be detected with a lesser number of bit errors than a low- power user at low SNR. At SNR of $9 \mathrm{~dB}$, the MMSE detection of high power is achieved without any bit errors. However, due to the presence of higher interference, the signal power is insufficient for the low power user to detect successfully. It is also evident that the bit errors incurred by the SIC detection of low power user depends on the MMSE estimation of the high-power user.

This is due to the fact that the successful SIC detection of low power user is possible only when the perfect detection of the high-power user using MMSE and the subsequent interference cancellation is accomplished. For code rate of $7 / 64$, SNR of $18 \mathrm{~dB}$ is sufficient enough to detect both the users without any bit errors.

The bit error performance of user- 1 which is estimated with the NOMA specific detection algorithm is further evaluated by encoding the 56 bits user data into $64,128,256,512,1024$ bits. This results in different code rates as shown in figure 6.

\section{Bit error performance analysis for polar coding} with different code rates

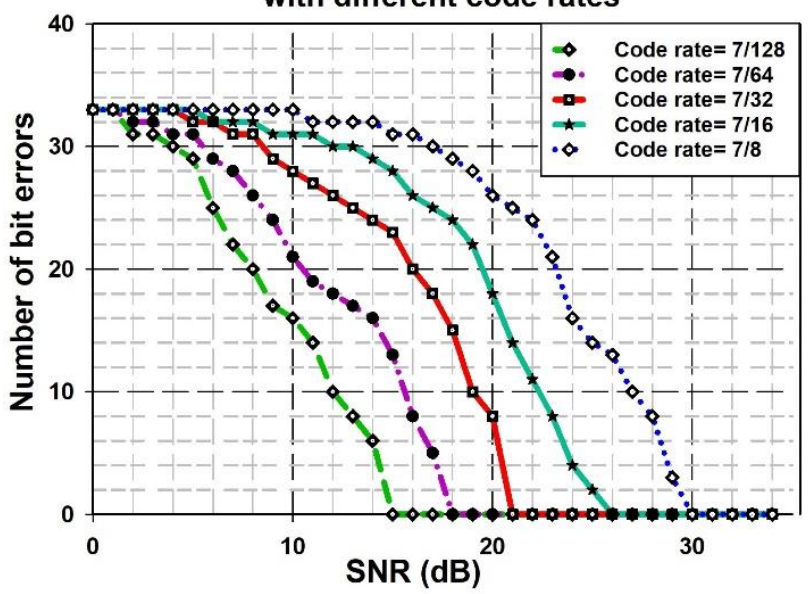

Figure 6. Bit errors obtained for various code rates for user 1.

It is observed that the SIC performance degrades with an increase in the code rate. The transmitted bits are successfully recovered with an SNR of $15 \mathrm{~dB}, 18 \mathrm{~dB}, 21 \mathrm{~dB}, 25 \mathrm{~dB}, 30 \mathrm{~dB}$ for code rates of 7/128,7/64,7/32,7/16, 7/8 respectively for 16-QAM modulation.

\begin{tabular}{|c|c|}
\hline TABLE I. & SIMULATION PARAMETERS \\
\hline Block & Comments \\
\hline User data & 56 bits \\
\hline Channel encoding & Polar coding \\
\hline Modulation & 16-QAM \\
\hline Precoder & Hadamard Transform \\
\hline Subcarrier mapping & $\begin{array}{l}32 \text { Resource blocks } \\
2 x \text { null subcarriers } \\
\text { localized mapping }\end{array}$ \\
\hline Transmit filter & $\begin{array}{l}\text { Root-raised cosine filter } \\
\text { Roll off factor: } 0.22 \\
\text { Filter length:5 } \\
\text { Oversampling factor: } 8\end{array}$ \\
\hline Power allocation & $\begin{array}{l}\text { UE-1: } 0.2 \times \mathrm{P} \text { total } \\
\text { UE-2: } 0.8 \times \mathrm{P} \text { total }\end{array}$ \\
\hline FFT size & 4098 \\
\hline Cyclic prefix & CP length-(1/4) OFDM symbol period \\
\hline MIMO & 2x2 MU- MIMO \\
\hline Channel model & Rayleigh with AWGN \\
\hline Receiver & MMSE-SIC \\
\hline
\end{tabular}

\section{CONCLUSION}

NOMA enables the users within a particular geographical region to be paired and jointly utilize the resources in non- orthogonal manner. Due to the non-constant envelope waveform adopted by OFDM, it suffers from high PAPR. By employing HTP NOMA, PAPR can be reduced by $3 \mathrm{~dB}$ than that of the non precoded NOMA. The link level performance of NOMA is evaluated for different SNR signifies that the SIC detection performance depends on the MMSE detection accuracy of high-power user.

There exist immense opportunities in designing least complex receivers, interference cancelling algorithms, optimized power allocation schemes and optimal user pairing for improved sum rates to enable NOMA as future wireless radio access technique.

\section{REFERENCES}

[1] 3GPP TR 38.812, "Study on Non-Orthogonal Multiple Access (NOMA) for NR," V16.0.0, (Release 16), December 2018.

[2] Islam, SM Riazul, et al. "Power-domain non-orthogonal multiple access (NOMA) in 5G systems: Potentials and challenges." IEEE Communications Surveys \& Tutorials 19.2 (2016): 721-742.

[3] Wang, Qi, et al. "Non-orthogonal multiple access: A unified perspective." IEEE Wireless Communications 25.2 (2018): 10-16.

[4] Zhang, Zekun, Haijian Sun, and Rose Qingyang Hu. "Downlink and uplink non-orthogonal multiple access in a dense wireless network." IEEE Journal on Selected Areas in Communications 35.12 (2017): 2771-2784

[5] Ding, Zhiguo, Fumiyuki Adachi, and H. Vincent Poor. "The application of MIMO to non-orthogonal multiple access." IEEE Transactions on Wireless Communications 15.1 (2015): 537-552.

[6] Benjebbour, Anass, and Yoshihisa Kishiyama. "Combination of NOMA and MIMO: Concept and Experimental Trials." 2018 25th International Conference on Telecommunications (ICT). IEEE, 2018.

[7] Zeng, Ming, et al. "Energy-efficient power allocation in uplink mmwave massive MIMO with NOMA." IEEE Transactions on Vehicular Technology 68.3 (2019): 3000-3004.

[8] Baig, Imran, et al. "On the PAPR Reduction: A Novel Filtering Based Hadamard Transform Precoded Uplink MC-NOMA Scheme for 5G Cellular Networks." 2018 1st International Conference on Computer Applications \& Information Security (ICCAIS). IEEE, 2018.

[9] 3GPP TS 38.212, "5G; NR; Multiplexing and channel coding", V 15.2.0 (Release 15), July 2018. 